\title{
O preparo do terço cervical e médio na endodontia contemporânea
}

\author{
Preparation of cervical and middle thirds in contemporary endodontics
}

\author{
Marcella Andrade Brito ${ }^{1 *}$, Danilo Barral de Araújo², Mônica Cardoso da Matta ${ }^{3}$ \\ ${ }^{1}$ Graduada em Odontologia pela Escola Bahiana de Medicina e Saúde Pública - EBMSP; ${ }^{2}$ Professor Associado de \\ Bioquímica Oral do Instituto de Ciências da Saúde. Universidade Federal da Bahia-UFBA; ${ }^{P}$ Professora Adjunto de \\ Odontologia Pré-Clínica em Endodontia e Estágio em Urgência. União Metropolitana de Educação e Cultura - UNIME
}

\begin{abstract}
Resumo:
Introdução: o tratamento endodôntico representa a terapia que trata as doenças pulpares e periapicais. O sucesso desse tratamento pode estar relacionado com o preparo do terço cervical e médio e a sua realização ou não contempla uma das vertentes da Endodontia contemporânea. Objetivo: identificar a prevalência de endodontistas que realizam o preparo cervical e médio, assim como as principais repercussões dentárias causadas por essa etapa, relacionando-as com instrumentos elencados neste estudo. Metodologia: trata -se de uma pesquisa observacional transversal que avalia, através de formulário online, a prevalência de endodontistas que realizam o pré-alargamento cervical e médio. O formulário foi enviado entre os dias 1 e e 30 de junho de 2021, pela plataforma Google Forms. A amostra foi composta por 102 endodontistas de ambos os gêneros. O formulário apresenta questões de gênero, idade e questionamento sobre tempo de formação e trabalho. Resultados: verificou-se que 93,1\% dos entrevistados alegaram realizar o preparo cervical e médio e a maioria, 67 respondentes, não acreditam que ele tem relação com o enfraquecimento dental. Para tal procedimento, a instrumentação rotatória é a mais utilzada com $54,9 \%$. Conclusão: o preparo cervical e médio é uma etapa operatória presente na maioria dos tratamentos endodônticos e sua realização pode estar relacionada com o enfraquecimento dental, no entanto, isso não promove o insucesso do tratamento e, de acordo com os entrevistados, os instrumentos rotatórios são os mais utilizados para essa etapa.
\end{abstract}

Palavras-Chave: Endodontia. Cavidade pulpar. Instrumentação. Doenças periapicais.

\begin{abstract}
Introduction: Endodontic treatment represents therapy that treats pulp and periapical diseases. The success of this treatment may be related to the preparation of the cervical and middle thirds and its realization or not contemplates one of the aspects of contemporary Endodontics. Objective: identify the prevalence of endodontists who perform cervical and middle preparation, as well as the main dental repercussions caused by this stage, relating them to the instruments listed in this study. Methodology: this is a cross-sectional observational study that assesses, through an online form, the prevalence of endodontists who perform cervical and mid-swelling pre-enlargement. The form was sent between June 1st and June 30th, 2021, by the Google Forms platform. The sample consisted of 102 endodontists of both genders. The form presents issues of gender, age and questions about training and work time. Results: it was found that 93.1\% of respondents claimed to perform cervical and middle preparation and the majority, 67 respondents, do not believe that it is related to dental weakening. For this procedure, rotary instrumentation is the most used with 54.9\%.Conclusion: cervical and middle preparation is an operative step present in most endodontic treatments and its performance may be related to dental weakening, however, this does not promote treatment failure and, according to respondents, rotary instruments are the most used for this step.

Keywords: Endodontics. Pulp Cavity. Instrumentation. Periapical Diseases.
\end{abstract}

\section{INTRODUÇÃO}

O tratamento endodôntico representa a terapia que melhor se relaciona com as doenças que envolvem os tecidos pulpares e periapicais, visto que tem a finalidade de promover a limpeza e a modelagem do canal radicular, dando possibilidades para que o elemento dental se mantenha em condições funcionais e estéticas na cavidade bucal (CHUGAL et al., 2017; MOORE et al., 2016).

Correspondente/Corresponding: *Marcella Andrade Brito - Universidade Federal da Bahia - UFBA - End: Rua Otacílio Santos, 241, City Park Brotas, Green Park, 501 - Tel: (77) 9910-7541 - E-mail: andrade. marcella@outlook.com
Tradicionalmente, o acesso coronário e o preparo químico-mecânico seguem princípios que permanecem inalterados por décadas. Definido como a etapa inicial, o acesso compreende a remoção completa do teto da câmara pulpar, seguido da localização dos orifícios de entrada dos canais radiculares; o preparo químico-mecânico, por sua vez, visa promover a limpeza do canal radicular com o objetivo de esvaziá-lo (LOPES; SIQUEIRA, 2016).

Previamente a essas duas etapas e contemplando princípios endodônticos tradicionais, o preparo do terço cervical e médio é realizado com a finalidade de remover as interferências localizadas na região cervical do canal radicular. Dessa forma, haverá um favorecimento na precisão da medida de comprimento do dente e um acesso 
mais direto dos instrumentos endodônticos ao ápice (SANTOS; SCARPARO, 2020).

Entretanto, sua realização tem sido associada a uma redução significativa da espessura dentinária, capaz de causar fraturas radiculares verticais (DUARTE, 2018). Visando a manutenção da dentina pericervical, principal responsável pela dissipação de forças provenientes da carga oclusal, acessos endodônticos minimamente invasivos (AEMI) têm sido propostos. Assim sendo, a preservação da dentina, somada à incompleta remoção do teto da câmara pulpar promovida pelo AEMI, pode aumentar a resistência mecânica dos dentes tratados endodonticamente e evitar as fraturas radiculares (BÜRKLEIN; SHÄFER, 2015; CHLUP et al., 2017; OZYUREK et al., 2018).

No entanto, esses acessos podem complexificar a localização dos canais e prejudicar o preparo químico-mecânico da raiz distal dos molares inferiores, por exemplo (NOSRAT et al., 2015; SILVA; SILVA, 2019,). Todavia, para o preparo químico-mecânico em acessos mais conservadores, é recomendada a utilização de sistemas reciprocantes. Esses sistemas permitem que todo tratamento endodôntico seja realizado com apenas um instrumento, dispensando a etapa do pré-alargamento cervical (KIEFNER; BAN; DE-DEUS, 2013). Além disso, sua característica de movimentação em sentido anti-horário promove uma modelagem e limpeza do canal radicular de forma mais segura (PLOTINO et al., 2017).

Isto posto, é notável que a realização do preparo cervical e médio contempla uma das vertentes que abrangem a Endodontia contemporânea. Contudo, não há na literatura científica muitos estudos a respeito dessa temática, motivo pelo qual esta pesquisa propõe evidenciar a prevalência de endodontistas que realizam o preparo cervical e médio, assim como as principais repercussões dentárias causadas por essa etapa, relacionando-as com os instrumentos elencados neste estudo.

\section{METODOLOGIA}

Trata-se de uma pesquisa observacional de característica transversal, para avaliar, por meio de formulário online, a prevalência de endodontistas que realizam o preparo do terço cervical e médio em seus tratamentos endodônticos. O formulário foi enviado entre os dias 1 은 e 30 de junho de 2021, pela plataforma Google Forms, tendo sido disponibilizado nas redes sociais Whatsapp, Instagram, Telegram. $\mathrm{O}$ termo de consentimento livre e esclarecido (TCLE) foi anexado e explicada a proposta da investigação, de modo que todos aqueles que concordassem estariam cientes do que se tratava. 0 projeto tem aprovação ética, pelo parecer de número 3.789.428.

Os dados foram tabulados em planilha Excel e posteriormente transferidos para o programa estatístico SPSS, versão 24 , para aplicação dos testes estatísticos. Realizou-se a tabela de frequência absoluta e relativa de todas as questões para apresentação dos dados pesquisados, referentes à idade e ao tempo de formação como cirurgião-dentista e como endodontista, considerando a média e o desvio padrão. Posteriormente, realizou-se o cruzamento de variáveis, sendo o tempo de formação como endodontista dicotomizado em $\leq 10$ anos de formação e > 10 anos de formação, além de se comparar com os questionamentos em relação ao preparo do terço cervical e médio. Também, reuniram-se todos aqueles que realizam o preparo do terço cervical e médio para comparação do tipo de instrumento utilizado, agrupando-se os instrumentos rotatórios e reciprocantes.

\section{RESULTADOS}

Analisaram-se 102 respostas de endodontistas, sendo $62,7 \%$ do sexo feminino e $37,3 \%$ do sexo masculino, ambos atuantes em prática clínica. Verificou-se que o tempo de formação médio correspondia a 12,75 anos $( \pm 7,471)$ para cirurgião-dentista, tendo atuação como especialista em endodontia 10,31 anos $( \pm 6,594)$. Em relação à realização do preparo do terço cervical e médio, $93,1 \%$ alegaram realizá-la, sendo que $92,2 \%$ afirmaram que tal etapa influencia diretamente no sucesso da terapia. A instrumentação rotatória foi a mais utilizada, representando $54,9 \%$ dos respondentes. Mais questões a respeito da realização do preparo do terço cervical e médio e seus efeitos no tratamento endodôntico podem ser vistos na Tabela 1.

Tabela 1 - Características da amostra e respostas a respeito do preparo do terço cervical e médio.

\begin{tabular}{|c|c|c|c|}
\hline $\begin{array}{c}\text { Preparo do terço cervical e } \\
\text { médio }\end{array}$ & & $\mathbf{n}$ & (\%) \\
\hline Idade & $36,07(7,335)$ & & \\
\hline $\begin{array}{l}\text { Por quanto tempo você exerce a } \\
\text { endodontia? }\end{array}$ & $10,31(6,594)$ & & \\
\hline \multirow{2}{*}{ Gênero } & Feminino & 64 & $(62,7)$ \\
\hline & Masculino & 38 & $(37,3)$ \\
\hline \multirow{2}{*}{$\begin{array}{l}\text { Em relação ao preparo do terço } \\
\text { cervical e médio, você o realiza? }\end{array}$} & Não & 7 & $(6,9)$ \\
\hline & Sim & 95 & $(93,1)$ \\
\hline \multirow{2}{*}{$\begin{array}{l}\text { Você acha que o preparo do terço } \\
\text { cervical e médio influencia no sucesso } \\
\text { da terapia endodôntica? }\end{array}$} & Não & 8 & $(7,8)$ \\
\hline & Sim & 94 & $(92,2)$ \\
\hline \multirow{4}{*}{$\begin{array}{l}\text { Com qual instrumento você realiza o } \\
\text { preparo do terço cervical e médio? }\end{array}$} & Gattes Glidden & 12 & $(11,8)$ \\
\hline & $\begin{array}{l}\text { Instrumentação } \\
\text { Reciprocante }\end{array}$ & 29 & $(28,4)$ \\
\hline & $\begin{array}{l}\text { Instrumentação } \\
\text { Rotatória }\end{array}$ & 56 & $(54,9)$ \\
\hline & Não realizo & 5 & $(4,9)$ \\
\hline \multirow{2}{*}{$\begin{array}{l}\text { Você acha que o preparo do terço } \\
\text { cervical e médio é responsável por um } \\
\text { grande índice de iatrogenia? }\end{array}$} & Não & 64 & $(62,7)$ \\
\hline & $\operatorname{Sim}$ & 38 & $(37,3)$ \\
\hline \multirow{2}{*}{$\begin{array}{l}\text { Você acha que o preparo do terço } \\
\text { cervical e médio traz repercussões às } \\
\text { unidades dentárias? }\end{array}$} & Não & 38 & $(37,3)$ \\
\hline & Sim & 64 & $(62,7)$ \\
\hline \multirow{2}{*}{$\begin{array}{l}\text { Você acha que o preparo do terço } \\
\text { cervical e médio traz um desgaste } \\
\text { desnecessário à essas regiões nas } \\
\text { unidades dentárias? }\end{array}$} & Não & 83 & $(81,4)$ \\
\hline & Sim & 19 & $(18,6)$ \\
\hline
\end{tabular}

Fonte: Dados da pesquisa. 
A variável tempo de atuação como endodontista de forma dicotomizada e as variáveis sobre o preparo do terço cervical e médio foram utilizadas para verificar as respostas, de acordo com a experiência clínica. Para aqueles que tinham $\leq 10$ anos de atuação, $96,9 \%$ responderam que realizam o preparo cervical e médio; e os que tinham $>10$ anos de atuação $86,5 \%$, também afirmaram realizá-lo. A respeito do sucesso da terapia endodôntica, dos que tinham $\leq 10$ anos $93,8 \%$ responderam que têm relação com o preparo do terço cervical e médio; e aqueles com $>10$ anos, $89,2 \%$ concordaram com isso. Sobre o preparo do terço cervical e médio e as repercussões nas unidades dentárias, $73 \%$ dos que afirmaram ter $>10$ anos de atuação responderam que esse preparo traz repercussões às unidades dentárias, sendo que mais detalhes podem ser encontrados na Tabela 2. E, quando o enfraquecimento dental foi relacionado com a etapa operatória estudada, concluiu-se que a maioria, 67 respondentes, realizam essa etapa porém não acredita que ela tenha relação com o enfraquecimento do dente (Tabela 3 ).

Tabela 2 - Atuação na Endodontia

\begin{tabular}{|c|c|c|c|}
\hline & & $\leq 10$ & $>10$ \\
\hline & & $n \%$ & $\mathrm{n} \%$ \\
\hline \multirow{2}{*}{$\begin{array}{l}\text { Em relação ao preparo do terço } \\
\text { cervical e médio, você o realiza? }\end{array}$} & Não & $02(3,1)$ & $05(13,5)$ \\
\hline & Sim & $63(96,9)$ & $32(86,5)$ \\
\hline \multirow{2}{*}{$\begin{array}{l}\text { Você acha que o preparo do terço } \\
\text { cervical e médio influenciano sucesso } \\
\text { da terapia endodôntica? }\end{array}$} & Não & $04(6,2)$ & $04(10,8)$ \\
\hline & Sim & $61(93,8)$ & $33(89,2)$ \\
\hline \multirow{2}{*}{$\begin{array}{l}\text { Você acha que o preparo do terço } \\
\text { cervical e médio é responsável por um } \\
\text { grande índice de iatrogenia? }\end{array}$} & Não & $41(63,1)$ & $23(62,2)$ \\
\hline & Sim & $24(36,9)$ & $14(37,8)$ \\
\hline \multirow{2}{*}{$\begin{array}{l}\text { Você acha que o preparo do terço } \\
\text { cervical e médio traz repercussões às } \\
\text { unidades dentárias? }\end{array}$} & Não & $28(43,1)$ & $10(27,0)$ \\
\hline & Sim & $37(56,9)$ & $27(73,0)$ \\
\hline \multirow{2}{*}{$\begin{array}{l}\text { Você acha que o preparo do terço } \\
\text { cervical e médio traz um desgaste } \\
\text { desnecessário à essas regiões nas } \\
\text { unidades dentárias? }\end{array}$} & Não & $56(86,2)$ & $27(73,0)$ \\
\hline & Sim & $09(13,8)$ & $10(27,0)$ \\
\hline
\end{tabular}

Fonte: Dados da pesquisa.

Tabela 3-Preparo do terço cervical e médio e o enfraquecimento dental.

\begin{tabular}{lccc}
\hline & & $\begin{array}{l}\text { Para você, o preparo do terço } \\
\text { cervical e médio tem relação } \\
\text { com o enfraquecimento dental } \\
\text { após a endodontia? }\end{array}$ \\
\cline { 2 - 3 } & Não & Sim \\
\hline $\begin{array}{l}\text { Em relação ao preparo do Não } \\
\text { terço cervical e médio, você } \\
\text { o realiza? }\end{array}$ & 1 & 6 \\
\hline
\end{tabular}

Fonte: Dados da pesquisa.

\section{DISCUSSÃO}

O preparo do terço cervical e médio, etapa prévia à modelagem do conduto, pode ser definida como uma técnica de ampliação do diâmetro no terço cervical e médio do canal radicular. Um dos principais objetivos é dar condições para os instrumentos endodônticos atuarem no interior do conduto, respeitando os princípios biológicos que norteiam a terapia endodôntica (SANTOS; SCAPARO, 2020).

Nesse contexto, os resultados deste estudo trouxeram a presença marjoritária dessa etapa operatória nos tratamentos endodônticos dos 102 endodontistas entrevistados, uma vez que, tanto aqueles que tinham $\leq 10$ anos de atuação $(96,9 \%)$, como os que tinham $>10$ anos $(86,5 \%)$, afirmaram realizar o preparo do terço cervical e médio. Além disso, sobre o sucesso da terapia para aqueles tinham $\leq 10$ anos de atuação, $93,8 \%$ responderam que o preparo do terço cervical e médio influencia no tratamento; e os que tinham $>10$ anos, $89,2 \%$ também concordaram com essa relação. Em consoância com esse resultado, Royer, Cord e Melo (2015), em seu trabalho, mostraram que o pré-alargamento cervical proporciona mais segurança na aferição do comprimento de trabalho durante a odontometria e minimiza o risco de flare-ups ao remover restos necróticos.

Entretanto, o pré-alargamento cervical e médio deve ser feito de maneira controlada, pois sua realização pode estar relacionada com a presença de paredes radiculares muito finas que alteram a proporcionalidade e aumentam a chance de fraturas radiculares. Portanto, o diâmetro do canal após o desgaste não deve ultrapassar um terço da largura da raiz (DUARTE, 2018). A respeito disso, na presente pesquisa, quando questionado sobre a relação do preparo do terço cervical e médio e as repercussões nas unidades dentárias, $73 \%$ dos que afirmaram ter $>10$ anos de atuação responderam que esse procedimento traz repercussões aos dentes. Porém, o enfraquecimento dentário, segundo 67 respondentes, não é uma repercussão que se relaciona com a etapa operatória estudada.

Ainda vale salientar que Tumenas et al. (2014) concluíram que, apesar da perda estrutural ocasionada pelo tratamento endodôntico, mediante a utilização do microscópio operatório, essa abordagem convencional pode ser desconstruída, pois essa tecnologia permite que as etapas do tratamento sejam realizadas preservando o máximo de estrutura dentária possível (TUMENAS et al., 2014). Ademais, a realização do pré-alargamento cervical reduz a tensão sobre o instrumento endodôntico e torna a terapia mais segura (MANIGLIA-FERREIRA et al., 2017).

É importante notar, portanto, que os instrumentos endodônticos estão intimamente relacionados com a quantidade de tecido dentinário removido e, por isso, o mercado dispõe de uma varidade de sistemas e de limas. As brocas Gates-Glidden, por exemplo, tradicionalmente representavam uma das mais utilizadas, porém, elas podem aumentar a chance de trincas na dentina, quando 
comparadas aos sistemas NiTi (Níquel-Titânio) (SILVA et al., 2020).

Mas, no que diz respeito a espessura da dentina cervical e a área do canal removida em molares inferiores, Duarte et al. (2011) mostraram que as limas rotatórias Orifice Shaper são as mais adequadas, pois, quando comparadas a LA Axxess 20.06 e Gates Glidden \#3, constituem o instrumento que deixa uma maior espessura de dentina, devendo-se ter cuidado ao utilizar as Gates Glidden \# 3 e LA Axxess 35.06 (ARSLAN et al., 2014; DUARTE et al., 2011). Além disso, essas limas rotatórias têm uma elevada flexibilidade e uma capacidade de memória elástica que diminui sua chance de fratura (GU et al., 2017; ROYER; CORD; MELO, 2015). Confirmando o que é descrito na literatura citada, os endodontistas participantes desta pesquisa, represenados por $54,9 \%$, optaram pela instrumentação rotatória como a preferida.

Além destes, o sistema reciprocante também tem sido utilizado na endodontia e apresenta um grande benefício, descoberto por Yared (2011): a possibilidade de realizar todo tratamento com apenas uma única lima, em sentido anti-horário. Dessa maneira, o tempo de trabalho será reduzido e a modelagem e limpeza do canal radicular serão feitas de forma mais segura, já que haverá um menor stress por torção (YARED, 2011). No entanto, Sisti (2017) observou que esses instrumentos, quando comparados aos rotatórios, promovem uma maior extrusão de microorganismos, capaz de proporcionar inflamação, infecção e dor pós-operatória, retardando a cicatrização apical (BORGES et al., 2016, NEVARES et al., 2015, SISTI, 2017, TOPÇUOĞLU et al., 2016).

Por fim, é importante destacar que, independente do sistema utilizado para a preparação do terço cervical e médio, essa etapa não deve ser negligenciada, pois diminui a possibilidade de acidentes operatórios, promove maior precisão ao aferir o comprimento do canal, reduz a extrusão de detritos para além do ápice e permite o acesso livre dos instrumentos pelo conduto radicular, minimizando o risco de fratura ocasionado pela lima e proporciando um melhor prognóstico aos dentes tratados endodônticamente (DUARTE, 2018).

\section{CONCLUSÃO}

A presente pesquisa concluiu que o preparo do terço cervical e médio é uma etapa operatória presente na maioria dos tratamentos endodônticos. Os dados avaliados mostraram que sua realização pode promover um desgaste excessivo, no entanto, o sucesso dos dentes tratados endodonticamente não é comprometido. Ademais, de acordo com este estudo, dentre os instrumentos dispostos no mercado a instrumentação rotatória é a mais indicada e utilizada pelos endodontistas, uma vez que mantém maior estrutura dental durante a etapa operatória.

\section{REFERÊNCIAS}

ARSLAN, H. et al. Efeito do ProTaper universal, Endoflare, Revo-S, instrumentos de alargamento coronal HyFlex e portões Glidden brocas na formação de fissuras. J. Endod., Cuiabá, v. 40, n. 10, p.1681-1683, 2014.

BORGES, A. H. et al. The influence of cervical preflaring on the amount of apically extruded debris after root canal preparation using different instrumentation system. J. Endod., Cuiabá, v. 42, n.3, p. 465-9, 2016.

BÜRKLEIN, S.; SCHÄFER, E. Minimally invasive endodontics. Quintessence Int., Münster, v. 46, n. 2, p. 119-124, Feb. 2015.

CHLUP, Z. et al. Fracture behaviour of teeth with conventional and miniinvasive access cavity designs. Journal of the European Ceramic Society, Barking, v. 37, n. 14, p. 4423-4429, 2017.

CHUGAL, N. et al. Endodontic treatment outcomes. Dent. Clin. North Am., v. 61, n. 1, p. 59-80, 2017.

SILVA, P. B. da et al. Influence of cervical preflaring and root canal preparation on the fracture resistance of endodontically treated teeth. BMC Oral Health, Porto Alegre, v. 20, p. 1-7. 2020.

DUARTE, M. A. et al. Efeitos de gates-Glidden, LA Axxess e brocas modeladoras de orifício na espessura da dentina cervical e área do canal radicular de molares inferiores. Braz. Dent. J., Bauru, v. 22, n.1, p. 28-31. 2011.

DUARTE, S. F. Influência do preparo cervical e preparo do canal radicular na resistência a fratura de dentes endodonticamente tratados: um estudo micro ct. 2018. 33f. Trabalho de Conclusão de Curso (Especialização) - Faculdade de Odontologia, Universidade do Rio Grande do Sul, Porto Alegre, 2018.

GU Y. et al. Vários instrumentos rotativos de níquel e titânio tratados ermicamente avaliados em $\mathrm{S}$ - canais de resina simulados em forma. J. Dent. Sci., [s.I.], v. 12, n. 1, p. 14-20, 2017. DOI: 10.1016/j. jds.2016.04.006.

LOPES, H. P.; SIQUEIRA, J. F. Jr. Endodontia, biologia e técnica. 4. ed. Rio de Janeiro: Elsevier, 2015. 1800p.

KIEFNER, P.; BAN, M.; DE-DEUS, G. Is the reciprocating movement per se able to improve the cyclic fatigue resistance of instruments? Int. Endod. J., Rio de Janeiro, v. 47, p. 430-436, July 2014.

MANIGLIA-FERREIRA, P. et al. Influence of reuse and cervical preflaring on the fracture strength of reciprocating instruments. Eur. J. Dent., Fortaleza, v. 11, n. 1, p. 041-047, 2017.

MOORE, B. et al. Impacts of contracted endodontic cavities on instrumentation efficacy and biomechanical responses in maxillary molars. J. Endod., New York, v. 42, n. 12, p. 1779-1783, 2016.

NEVARES, G. et al. Apical extrusion of debris produced during continuous rotating and reciprocating motion. Scientific World Journal, [s.I.], v. 2015, 2015.

NOSRAT, A. et al. Middle mesial canals in mandibular molars: incidence and related factors. J. Endod., New York, v. 41, n.1, p. 28-32, 2015.

OZYUREK, T. et al. The effects of endodontic access cavity preparation design on the fracture strength of endodontically treated teeth: Traditional versus conservative preparation. J. Endod., New York, v. 44, n. 5, p. 800-805, 2018.

PLOTINO, G. et al. Fracture strength of endodontically treated teeth with different access cavity designs. J. Endod., New York, v. 43, n. 6, p. 995-1000. 2017. 
ROYER, J.; CORD, C. B.; MELO, T. F. de Análise de quatro instrumentos para preparo cervical do canal quanto à área e à espessura de desgaste na região de Furca. Odonto, Caxias do Sul, v. 23, n. 45-46, p.37-45. 2015.

SANTOS, R. B.; SCARPARO R. K. Endodontia pré-clínica. Porto Alegre: Evangraf, 2020. 138p.

SILVA, P. A. C.; SILVA, I. S. N. Minimally invasive endodontical acess: literature review. SALUVIS-TA, Bauru, v. 38, n. 1, p. 195-212, Jan. 2019.

SISTI, I. C. G. Extrusão de debris pelo forame apical durante o tratamento endodôntico. 2017. 27 f. Trabalho de Conclusão de Curso (Especialização) - Universidade Estadual de Campinas, Piracicaba,
2017. 27 p.

TOPÇUOĞLU H. S. et al. Effect of coronal flaring on apical extrusion of debris during root canal instrumentation using single-file systems. Int. Endod. J., Oxford, v. 49, n. 9, p. 884-889, Sept. 2016.

TUMENAS, I. et al. M. Odontologia minimamente invasiva. Rev. Assoc. Paul. Cir. Dent., São Paulo, v. 68, n. 4, p. 285-95. 2014.

YARED, G. Canal preparation using one reciprocating instrument without prior hand filing: A new concept. International Dentistry, Ontario, v. 2, n. 2, p 1-7. 2011.

Submetido em: 19/11/2021

Aceito em: 20/11/2021 